\title{
Guidelines of the Italian Society of Videosurgery in Infancy for the minimally invasive treatment of the ureteropelvic-junction obstruction
}

\author{
Salvatore Fabio Chiarenza, ${ }^{1}$ Cosimo Bleve, ${ }^{1}$ Ciro Esposito, ${ }^{2}$ Maria Escolino, ${ }^{2}$ Fabio Beretta, ${ }^{3}$ \\ Maurizio Cheli, ${ }^{4}$ Vincenzo Di Benedetto, 5 Maria Grazia Scuderi, 5 Giovanni Casadio, 6 Maurizio Marzaro, ${ }^{7}$ \\ Leon Francesco Fascetti,8 Pietro Bagolan,9 Claudio Vella,10 Maria Luisa Conighi,1 Daniela Codric,,11 \\ Simona Nappo, 12 Paolo Caione ${ }^{1}$ \\ 1Pediatric Surgery Unit, Local Health Unit 8, S. Bortolo Hospital, Vicenza; 2Pediatric Surgery Unit, Federico II \\ University, Naples; ${ }^{3}$ Pediatric Surgery Unit, S. Chiara Hospital, Trento; ${ }^{4}$ Pediatric Surgery Unit, Papa Giovanni XXIII \\ Hospital, Bergamo; ${ }^{5}$ Pediatric Surgery Unit, Vittorio Emanuele Hospital, Catania; ${ }^{6}$ Pediatric Surgery Unit, Parma \\ University Hospital, Parma; ${ }^{7}$ Pediatric Surgery Unit, Local Health Unit 2, Treviso Hospital; ${ }^{8}$ Pediatric Surgery Unit, \\ Padua University Hospital, Padua; ${ }^{9}$ Neonatal Surgery Unit, Pediatric Hospital Bambino Gesù, Rome; 10 Pediatric \\ Surgery Unit, Children Hospital V. Buzzi, Milan; 11Pediatric Surgery Unit, IRCCS Burlo Garofolo, Trieste; 12 Pediatric \\ Surgery and Urologic Unit, Pediatric Hospital Bambino Gesù, Rome, Italy
}

\section{Linee guida della Società Italiana di Videochirurgia Infantile}

Le linee guida della Società Italiana di Videochirurgia Infantile (SIVI) sono uno strumento semplice e pratico redatto ed approvato da consiglio direttivo della società. Sono frutto di un lungo lavoro di revisione sistematica della letteratura rivisto ed integrato dal parere di esperti nel campo della Chirurgia Mininvasiva Pediatrica. Esse sono intese ad aiutare il chirurgo pediatra che si avvicina o già pratica la chirurgia Mininvasiva; le linee guida della SIVI hanno lo scopo di facilitare la scelta dell'indicazione e del trattamento chirurgico ottimale per le principali patologie chirurgiche pediatriche. Tuttavia, vista la complessità delle malformazioni congenite, delle patologie chirurgiche pediatriche e l'estrema varietà dell'ambiente sanitario, è importante sottolineare come l'approccio suggerito potrebbe non essere necessariamente l'unico approccio accettabile e/o percorribile. Le linee guida devono essere considerate uno stru-

\footnotetext{
Correspondence: Salvatore Fabio Chiarenza, Pediatric Surgery Unit, Local Health Unit 8, S. Bortolo Hospital, viale Rodolfi 37, 36100 Vicenza, Italy.

Tel: +39.0444.75.2642 - Fax:+39.0444.75.2643.

Email: fabio.chiarenza@ulssvicenza.it
}

Key words: SIVI; Minimally invasive treatment; Ureteropelvic-junction obstruction.

Project supervisors: Salvatore Fabio Chiarenza, Paolo Caione.

Received for publication: 13 September 2017.

Accepted for publication: 19 September 2017.

This work is licensed under a Creative Commons Attribution

NonCommercial 4.0 License (CC BY-NC 4.0).

(C) Copyright S.F. Chiarenza et al., 2017

Licensee PAGEPress, Italy

La Pediatria Medica e Chirurgica 2017; 39:174

doi:10.4081/pmc.2017.174 mento flessibile in quanto poi è il medico che, con il consenso dei genitori/tutori, avrà il compito di scegliere l'approccio più adatto al singolo paziente. Tale approccio sarà attuato in scienza e coscienza e in base alla patologia ed alle eventuali variabili esistenti al momento della decisione.

La massima accessibilità possibile è garantita dalla pubblicazione sulla rivista ufficiale della SIVI: La Pediatria Medica e Chirurgica.

Il progetto linee Guida è stato approvato dall'assemblea generale del Congresso SIVI 2016 di Madrid.

La revisione delle Linee Guida pubblicate è stata effettuata dal Consiglio Direttivo della SIVI e della Società Italiana di Urologia Pediatrica (SIUP).

Consiglio Direttivo SIVI

Presidente: Salvatore Fabio Chiarenza (Vicenza)

Vice Presidente: Paolo Caione (Roma)

Segretario: Maria Grazia Scuderi (Catania)

Tesoriere: Maurizio Marzaro (Treviso)

Consiglieri:

Maria Escolino (Napoli)

Francesco Fascetti Leon (Padova)

Daniela Codrich (Trieste)

Pietro Bagolan (Roma)

Maurizio Cheli (Bergamo)

Revisori dei Conti:

Cosimo Bleve (Vicenza)

Giovanni Casadio (Parma)

Probi Viri:

Claudio Vella (Milano)

Fabio Beretta (Trento)

Web Master: Ciro Esposito (Napoli)

Consiglio Direttivo SIUP

Presidente: Vincenzo di Benedetto (Catania)

Vice Presidente: Nicola Capozza (Roma)

Past President: Waifro Rigamonti (Trieste)

Segretario: Vincenzo Bagnara (Catania)

Tesoriere: Giovanni Mosiello (Roma) 


\section{Consiglieri:}

Marco Castagnetti (Padova)

Massimo Catti (Torino)

Maria Grazia Scarpa (Trieste)

Giorgio Giuseppe Selvaggio (Milano)

Revisori dei Conti:

Francesco Battaglino (Vicenza)

Simona Gerocarni Nappo (Roma)

\section{Introduzione}

Le linee-guida cliniche per l'idronefrosi hanno lo scopo di indirizzare nel miglior modo l'approccio nella diagnosi e nel trattamento di questa importante e frequente patologia congenita dell'età pediatrica ed evolutiva, che ha visto recentemente profonde innovazioni dell'iter diagnostico e ancor più del trattamento. Oggi sempre più frequente è il ricorso a trattamenti innovativi mini-invasivi con tecniche video-laparoscopiche, di cui la SIVI è ferma sostenitrice. Queste linee-guida sono sviluppate per essere uno strumento flessibile, offerto allo specialista chirurgo-urologo della SIVI per selezionare l'approccio più adatto al singolo paziente fra le molteplici variabili presenti nella letteratura più aggiornata. Esse sono preparate da un Gruppo di Lavoro ristretto e approvate dal Comitato Direttivo della SIVI.

\section{Inquadramento clinico e epidemiologia}

L'idronefrosi, caratterizzata da dilatazione del sistema pielocaliceale renale con possibile compromissione funzionale del parenchima renale, è la più comune anomalia congenita dell'apparato urinario riconosciuta in epoca prenatale tramite lo screening ecografico in gravidanza. Tuttavia è oggi ben noto che dilatazione pielo-caliceale non significa necessariamente ostruzione e sofferenza parenchimale. È inoltre da sottolineare che circa il $60 \%$ delle pielectasie riconosciute in utero tendono a risolvere spontaneamente nei primi mesi o anni dalla nascita. È stato calcolato che circa meno del 5\% delle idronefrosi osservate in gravidanza hanno poi una vera ostruzione della giunzione pielo-ureterale che richiede intervento di pieloplastica. Oltre alla diagnosi con screening ecografico prenatale o neonatale, i sintomi di presentazione nei bambini di età maggiore sono abitualmente dolori addominali come lombalgia o coliche oppure infezioni urinarie.

\section{Definizione}

L'ostacolo al passaggio anterogrado delle urine attraverso la giunzione pielo-ureterale (GPU) provoca abitualmente un incremento talora progressivo del sistema pielo-caliceale intrarenale, con uno spettro molto variabile di riduzione della funzione parenchimale alla reno-scintigrafia. Non raramente la funzione renale si mantiene normale per lungo periodo, talora l'ostruzione è così serrata da determinare perdita completa della funzionalità del rene affetto.

I meccanismi ezio-patogenetici che possono essere alla base dell'ostruzione del GPU possono essere più di uno: segmento adinamico e ipoplastico del giunto superiore e del tratto prossimale dell'uretere; lembi valvolari; inserzione alta dell'uretere nella pelvi; uretere prossimale tortuoso (uretere a cavaturacciolo); vasi polari inferiori aberranti (ostacolo estrinseco alla via escretrice).
Nel 5-10\% dei casi con ostruzione del GPU si può associare una seconda anomalia dell'apparato urinario, quale reflusso vescico-ureterale (RVU), ostruzione del giunto uretero-vescicale (GUV), megauretere primitivo non ostruttivo e non refluente omoo controlaterale, displasia renale multicistica controlaterale.

\section{Diagnosi}

Un importante aggiornamento dell'iter diagnostico si è consolidato nelle ultime 2-3 decadi nell'idronefrosi infantile, differenziandosi nettamente dalla diagnostica dell'idronefrosi nel paziente adulto.

\section{Esami ematochimici}

Specie nel caso d'interessamento renale bilaterale, esami ematochimici per funzionalità renale e in particolare creatininemia sono opportuni.

\section{Ecografia renale e apparato urinario}

Rappresenta la prima indagine morfologica, indipendentemente dall'età del paziente. L'ecografia nei primi 3-4 giorni di vita può falsamente rassicurare sul recupero dell'idronefrosi riconosciuta in utero, a causa della disidratazione del neonato con il calo ponderale post-natale. Pertanto si raccomanda di effettuare un esame ecografico dopo i primi 4-5 giorni di vita, abitualmente fra la 1' e la 2' settimana di età. I parametri ecografici più utili sono il diametro longitudinale renale, lo spessore parenchimale in sede meso-renale e soprattutto il diametro antero-posteriore (DAP) della pelvi all'ilo. Quest'ultimo è di gran lunga il parametro più significativo, necessario anche per il follow-up. Entrambi i reni vanno valutati, anche in proiezione posteriore. Attenzione va posta nel valutare l'eventuale dilatazione caliceale e la presenza o meno di dilatazione ureterale, mono o bilaterale. La presenza di un uretere dilatato omolaterale può comprendere differenti possibilità diagnostiche dalla ostruzione del GPU, RVU, ostruzione del GUV o megauretere primitivo.

\section{Cistouretrografia minzionale}

È utile per valutare la presenza eventuale di RVU o di ostacolo allo svuotamento vescicale, come in caso di valvole congenite dell'uretra posteriore. Va effettuata sotto profilassi antibatterica urinaria, con urine sterili. Tuttavia è indicata se sono presenti in ecografia ureteri dilatati o comunque visibili ecograficamente, vescica sovra distesa e/o a pareti ispessite, doppio distretto renale o rene singolo, reni iperecogeni anche dopo le prime settimane di vita.

Si ritiene oggi non necessario procedere a cistografia o cistosonografia in caso d'idronefrosi singola isolata, senza altri reperti ecografici patologici a carico delle vie urinarie. È questionabile l'indicazione alla cistografia in caso di idronefrosi bilaterale semplice, senza dilatazioni ureterali o altre anomalie urinarie riconoscibili ecograficamente.

\section{Scintigrafia renale sequenziale}

La scintigrafia renale sequenziale usando come radioisotopo il MAG3 (Mercapto-acetil-triglicina marcata con Tecnezio $99 \mathrm{~m}$ ), o più raramente con DTPA, è esame funzionale divenuto essenziale in età pediatrica per lo studio della attività renale e della fase di escrezione della radio urina, attraverso valutazione della captazione radio isotopica da parte dei reni e delle curve di drenaggio pielico. Nella fase iniziale di captazione è valutata la perfusione parenchimale e la funzione differenziale di ciascun rene che con- 
tribuisce in percentuale alla funzione renale globale. La fase di drenaggio pielico è rappresentata da una curva, la cui pendenza dopo la fase di uptake indica la velocità di deflusso pielo-ureterale. In caso di curva ridotta o assente per alterato drenaggio pielico, si aggiunge il test diuretico con furosemide $(0,5-1,0 \mathrm{mg} / \mathrm{kg})$, considerando un drenaggio insufficiente, cioè una ostruzione del giunto, quando il tempo di dimezzamento del radioisotopo nella regione di interesse pielo-renale $(\mathrm{T} / 2)$ è maggiore di 20 minuti. Tale esame, tuttavia, è in parte dipendente da vari fattori, quali lo stato di idratazione del bambino, la funzione renale e il riempimento vescicale, per cui esistono precise linee-guida della Società Europea di Medicina Nucleare Pediatrica. Lo studio renoscintigrafico è raccomandato idealmente dal $3^{\circ}$ mese di età, a maturazione renale completata, ma in casi selezionati può essere effettuato a partire dal primo mese di vita. L'uso in epoca neonatale offre una minore attendibilità.

\section{Scintigrafia renale statica}

La scintigrafia con Acido Dimercapto-succinico marcato con Tecnezio 99m viene utilizzata in caso di coesistente RVU, per verificare la presenza di lesioni cicatriziali renali, o in caso di rene unilaterale poco funzionante per decidere se procedere ad eventuale nefrectomia invece che intervento conservativo renale.

Un pronto iter diagnostico con le indagini su riportate si rende necessario nei piccoli bambini con reni ecograficamente iperecogeni, storia prenatale di oligo-idramnios, rene idronefrotico solitario, idronefrosi bilaterale severa (DAP $>20 \mathrm{~mm}$ ), o vescica abnorme o a pareti ispessite, come in caso di valvole congenite dell'uretra posteriore. Queste situazioni possono predisporre a danno renale progressivo e ad episodi di urosepsi a rischio anche della vita del piccolo paziente.

\section{Indicazioni per l'intervento}

L'intervento disostruttivo per il GPU è indicato quando è confermata idronefrosi con ostruzione al deflusso di urine. Classicamente vengono individuati come parametri predittivi di ostruzione una funzione renale differenziale inferiore al $40 \% \mathrm{e} / \mathrm{o}$ una curva renoscintigrafica di eliminazione piatta o in incremento con T/2 superiore a 20 min'. A livello ecografico è considerato significativo un progressivo incremento del DAP pielico o un DAP maggiore di $30 \mathrm{~mm}$ con calici dilatati (rischio intervento pari al 90\%). Indicazioni cliniche all'intervento sono anche le infezioni urinarie e ricorrente sintomatologia dolorosa. Particolare attenzione alla corretta indicazione chirurgica si deve porre nelle idronefrosi con pelvi intrarenale, o con notevole interessamento caliceale, nelle idronefrosi bilaterali, e nei reni solitari.

\section{Approcci chirurgici}

\section{Chirurgia a cielo aperto}

La Anderson-Hynes dismembered pyeloplasty o pieloplastica con resezione del GPU è forse tuttora considerata il gold standard, ma nuove tecniche mini-invasive video-laparoscopiche stanno rapidamente prendendo piede. La tecnica ha risultati positivi nel 95-98\% dei casi. Nei primi anni di vita viene abitualmente utilizzato un approccio retroperitoneale con incisione sottocostale o lombare posteriore sezione o meglio, splitting (divaricazione) dei muscoli della parete addominale, con accesso diretto alla loggia renale e al GPU. Si procede a resezione del giunto ed eventuale parte della pelvi eccedente, a spatulazione longitudinale del tratto prossimale dell'uretere prossimale e quindi ad anastomosi pieloureterale a becco di flauto (sutura con 2 emicontinue o con punti staccati a lungo riassorbimento (calibro 5 o 60 7/zeri). Si può applicare oppure si può evitare l'uso di stent (cateteri) trans-anastomotico pielo-ureterale. Vari tipi di stent possono essere utilizzati, a scelta del chirurgo operatore: prevalentemente doppio J e stent pieloureterale nefrostomico sono i più utilizzati. Si può applicare nefrotomia transitoria decompressiva e si può o meno lasciare un drenaggio nella loggia renale. Abitualmente, ma non necessariamente, un catetere vescicale aiuta a tenere decompresse le vie urinarie superiori nei primi 2-3 giorni post-operatori. Questa tecnica è oggi spesso offerta con approccio mini-invasivo video-laparoscopico.

Altre tecniche di pieloplastica sono raramente utilizzate nel bambino: la Scardino-Prince, la plastica ad Y-V di Foley, la CulpDe Weerd e la Fenger-plasty (simile alla Y-V con incisione del giunto longitudinalmente risuturandolo trasversalmente tipo Heineke-Mikulicz. La uretero-calicostomia può essere utilizzata in condizioni particolari, come grossolana cicatrice a livello del GPU e idronefrosi in rene a ferro di cavallo. Prerequisito per il successo della uretero-calicostomia è l'escissione di buon tassello di parenchima per permettere buona anastomosi tra urotelio caliceale e quello dell'uretere prossimale.

\section{Chirurgia minimamente invasiva}

Comprende tecniche che recentemente stanno riscuotendo maggiore diffusione, soppiantando in buona parte le tradizionali tecniche open. Comprende gli approcci laparoscopici e retroperitoneoscopici al GPU, la pieloplastica assistita con singolo trocar, la procedura del vascular hitch laparoscopico e la pieloplastica robotassistita.

\section{Work-up preoperatorio}

Gli esami ematici abitualmente richiesti sono: emocromo, creatininemia, elettroliti, prove emogeniche e eventualmente gruppo sanguigno. Specie per le procedure laparoscopiche trans-peritoneali, è raccomandabile una preparazione intestinale con farmaci antifermentativi e lassativi e/o clistere evacuativo nelle $24 \mathrm{~h}$ precedenti l'intervento.

Il consenso chirurgico dovrebbe includere informazioni sulla diagnosi, sul tipo d'intervento proposto, sulle alternative, sulla possibile necessità di conversione. È bene informare su quali stent e cateteri saranno utilizzati e infine sulle possibili complicanze quali sanguinamento, perdita di urine, infezioni urinare e rischio di persistente ostruzione con possibile necessità di reintervento.

\section{Preparazione della sala operatoria per l'approccio laparoscopico trans peritoneale (pieloplastica e vascular hitch)}

Il paziente viene posto in posizione laterale o semi-laterale con il lato opposto a quello idronefrotico vicino al bordo del tavolo operatorio. Il chirurgo è di fronte al paziente, con la colonna videolaparoscopica alle spalle del paziente. Lo strumentario laparoscopico prevede abitualmente 2 porta-aghi, tampone montato, grasper e strumenti da presa, uncino, passafili, aspiratore-irrigatore, con elettrificazione mono e/o bipolare e possono variare a seconda della preferenza del chirurgo operatore.

\section{Preparazione della sala operatoria per l'approccio retro-peritoneoscopico}

Il paziente è posizionato in decubito laterale con un soffice cuscino o rotolo sotto il fianco contro laterale così da ampliare lo spazio tra arcata costale e cresta iliaca per il posizionamento delle 
porte laparoscopiche. Il chirurgo e l'assistente si posizionano alle spalle del paziente, la strumentista al loro fianco o ai piedi del paziente e la colonna video-laparo è dal lato opposto cioè verso l'addome. Gli strumenti laparoscopici sono analoghi a quelli dell'accesso trans-peritoneale.

\section{Tecnica chirurgica: pieloplastica laparoscopica}

Sono utilizzati abitualmente un trocar da 5 o $10 \mathrm{~mm}$ in sede ombelicale, eventualmente con palloncino (trocar di Hasson), per l'ottica da 5 o $10 \mathrm{~mm}$ (preferibilmente da $30^{\circ}$ ) e 2 porte da 3 o 5 $\mathrm{mm}$ per lo strumentario. Queste ultime sono da posizionare in modo da ottenere la corretta triangolazione degli strumenti laparoscopici. Talora si può rendere necessaria una $3^{\circ}$ porta operativa (esempio per sollevare 1'ala epatica nella pieloplastica destra o per sospendere l'anastomosi). Sul lato sinistro, più frequentemente è sufficiente una finestra trans-mesocolica per raggiungere lo spazio retroperitoneale e quindi effettuare la pieloplastica o il vascular hitch. Sul lato destro, può essere più spesso necessario mobilizzare la flessura epatica del colon per accedere alla loggia renale in corrispondenza del GPU. La pieloplastica secondo Anderson-Hynes a questo punto viene effettuata in maniera analoga alla procedura open, con resezione del giunto, spatulazione dell'uretere prossimale e anastomosi pielo-ureterale a becco di flauto. La sutura intracorporea richiedere solitamente un certo grado di expertise laparoscopica e può essere effettuata con 2 emi-continue o con punti staccati, utilizzando fili a lungo riassorbimento, usualmente $5 / 0$ o $6 / 0$, monofilamento o intrecciati. La applicazione dello stent interno pielo-ureterale JJ non è obbligatoria ma spesso utilizzata per migliore sicurezza nel drenaggio pielico post-operatorio immediato. Lo stent può essere inserito con varie metodiche, generalmente per via anterograda da una porta laparoscopica o per via percutanea su ago-cannula o talvolta anche per via cistoscopica. Al termine della procedura viene solitamente eseguita la chiusura della finestra peritoneale anche se non vi è evidenza scientifica a suo supporto. Nella loggia renale viene solitamente posizionato un drenaggio che viene fatto fuoriuscire da contro-apertura percutanea declive o dalla porta laparoscopica più distale.

\section{Tecnica chirurgica: pieloplastica retro-peritoneoscopica}

Nella gran pare dei casi, sono sufficienti due porte operative da 3 o da $5 \mathrm{~mm}$ e una per l'ottica da 5 o $10 \mathrm{~mm}$. L'ottica può essere da $0^{\circ}$ o da $30^{\circ}$ a seconda della preferenza dell'operatore. Una incisione cutanea di 8-12 mm è effettuata all'apice o al di sotto della 12 costa; per via smussa si accede al retro peritoneo, avendo lo psoas come guida e orientamento. Poiché il retroperitoneo è una cavità virtuale, per sviluppare un sufficiente spazio di lavoro (working space) attraverso la prima incisione (di lunghezza maggiore) vengono introdotte delle garze umide o più raramente un pallone gonfiato di aria secondo la tecnica di Gaur. Nei pazienti più grandicelli, la dissezione può avvenire digitalmente. Va posta molta attenzione a non procurare perforazioni sul peritoneo parietale per evitare perdite di gas all'interno del peritoneo con conseguente difficoltà a creare lo spazio di lavoro. Una volta creato sufficiente spazio retro peritoneale, il rene appare sospeso e attaccato al peritoneo verso l'alto e la pelvi appare inferiormente con l'uretere che decorre sullo psoas; vengono quindi introdotti 2-3 trocar operativi da 3 o $5 \mathrm{~mm}$. La GPU viene isolata per via smussa e quindi può essere effettuata una pieloplastica dismembered secondo Anderson-Hynes, rispettando i principi e i tempi chirurgici già descritti. Abitualmente la procedura video-laparoscopica retro peritoneale è più impegnativa per la ristrettezza del campo operatorio e richiede un livello alto di expertise in MIS. A livello tecnico, si identificano i seguenti passaggi, che possono variare a secon- da della esperienza e preferenza dell'operatore: A) punto di ancoraggio della giunzione sul muscolo psoas. B) apertura della pelvi dilatata in posizione declive e spatulazione dell'uretere prossimale. C) sutura del bordo anteriore dell'anastomosi pielo-ureterale (punti interrotti o continui). D) resezione della GPU e di parte della pelvi eccedente (non necessariamente). E) inserimento di tutore interno JJ per via anterograda percutanea. F) completamento dell'anastomosi sul bordo posteriore e della pieloplastica con sutura continua o interrotta. G) eventuale drenaggio della loggia renale.

\section{Tecnica chirurgica: pieloplastica assistita con singolo trocar}

È una tecnica che coniuga i vantaggi della mini-invasività degli approcci video-laparoscopici con la praticità e sicurezza di una pieloplastica a cielo aperto a livello cutaneo. È tuttavia utilizzabile con maggior sicurezza nei pazienti di ridotto peso corporeo (inferiore a $20 \mathrm{~kg}$ ), di età entro i 5-6 anni, con ridotto pannicolo adiposo e con pareti addominali relativamente esili. Le tappe principali della procedura prevedono: A) incisione cutanea di 15-18 $\mathrm{mm}$ all'apice o al di sotto della 12 costa ipsilaterale, come nella retroperitoneoscopia precedentemente descritta. B) Introduzione di trocar a palloncino di Hasson da $10 \mathrm{~mm}$ e ottica da $10 \mathrm{~mm}$ con canale operativo da $5 \mathrm{~mm}$. Strumentario laparoscopico da $5 \mathrm{~mm}$ analogo alle altre procedure video-laparoscopiche. C) Mobilizzazione per via smussa del peritoneo posteriore dal muscolo psoas fino alla loggia renale tramite ottica e tampone montato laparoscopico, favorito dalla pressione della $\mathrm{CO}_{2}$ a circa $9 \mathrm{~mm} / \mathrm{Hg}$. D) Identificazione del polo renale inferiore, dell'uretere prossimale e della pelvi, E) Sollevamento su vessel-loop del giunto pielo-ureterale fino al piano sottocutaneo. F) Rimozione del trocar operativo e prosecuzione della procedura secondo la classica AndersonHynes a livello cutaneo-sottocutaneo (loops o sistemi di ingrandimento ottico), avendo estrinsecato il GPU ostruito attraverso la porta laparoscopica. G) Inserito lo stent JJ e completata la sutura con fili 5 o 6/0, riposizionamento del giunto nella loggia renale e verifica laparoscopica di assenza di kinking o torsioni dell'asse escretore. H) Si può applicare o omettere il drenaggio della loggia. Utile foley vescicale per 24-72 ore.

La tecnica richiede un livello non elevato di esperienza laparoscopica, in quanto non sono previste suture intracorporee. Può essere utilizzata anche in caso di vasi aberranti, ma richiede comunque la sezione del giunto e la trasposizione uretero-vascolare. Non è utilizzabile comunemente per la vascular hitch. In caso di necessità, è agevole ampliare del necessario l'incisione della porta laparoscopica e procedere con una semi-conversione.

\section{Tecnica chirurgica: procedura del vascular hitch}

Questa tecnica si può utilizzare in casi di vasi renali polari inferiori aberranti e ostruenti l'uretere prossimale. Per utilizzare questa metodica è essenziale la corretta selezione del paziente: la vascular hitch è riservata alle idronefrosi da pura compressione estrinseca del giunto da vasi polari inferiori aberranti. È necessario sapere che la compressione estrinseca può coesistere con un'ostruzione intrinseca del GPU: tali casi vanno riconosciuti, in modo da evitare un fallimento a lungo termine della tecnica di vascular hitch qualora adottata. La tecnica è sicuramente attraente, in quanto non richiede sezione e anastomosi della via escretrice e non sistematica applicazione di stent pielo-ureterale.

La forma classica d'idronefrosi da vasi polari aberranti si presenta solitamente in bambini in età scolare o adolescenti con crisi dolorose a crisi e dilatazione pielica intermittente più frequentemente senza calicectasia. La presenza dei vasi aberranti può essere ricercata con Eco-color-doppler e con Angio-RM, anche se la certezza viene solo dall'esplorazione chirurgica. 
L'accesso laparoscopico trans-peritoneale è la prima scelta, in quanto i vasi decorrono anteriormente alla via escretrice, ed è analogo a quello della pieloplastica dismembered. Quindi, identificati i vasi aberranti, essi vengono delicatamente mobilizzati cranialmente sulla pelvi, in modo da liberare il giunto. Un test intraoperatorio alla furosemide $(1 \mathrm{mg} / \mathrm{kg})$ dopo carico idrico $(20$ $\mathrm{mL} / \mathrm{kg}$ ) risulta determinante per visualizzare laparoscopicamente il buon transito pielo-ureterale con decompressione pielica, escludendo pertanto la coesistenza di una ostruzione intrinseca. Il difficoltoso svuotamento della pelvi dopo test, l'aspetto di una giunzione sottile fibrotica e non lineare dovrebbe mettere in guardia il chirurgo dall'effettuare un vascular hitch invece che la classica Anderson-Hynes. Possono essere usate 2-3 porte operative da 3 o $5 \mathrm{~mm}$ e una porta ombelicale per l'ottica da 5 o 10 $\mathrm{mm}$. L'accesso alla pelvi renale è analogo a quello utilizzato per la pieloplastica. I vasi polari inferiori sono identificati, mobilizzati (eventualmente su fettuccia) e la pelvi con l'uretere prossimale sono adeguatamente liberati da eventuali aderenze. Il test da carico idrico con furosemide deve dimostrare buon passaggio di urine con decompressione pielica. In caso di dubbia risposta, può essere realizzata una pieloplastica con trasposizione ureterovascolare (dismembered secondo Anderson-Hynes) o senza trasposizione (es. Fenger-plasty). L'ancoraggio dei vasi polari in posizione più craniale e lontano dalla GPU viene solitamente realizzato con 2-3 suture staccate che fissino il tessuto perivascolare alla parete pielica secondo Hellstrom (1949), o creando una amaca con la parete pielica, a sostegno del peduncolo vascolare, proposta da Chapman (1959) con 2-4 punti di sutura 2,3 o 4/0 di materiale riassorbibile o non riassorbibile. Quest'ultima tecnica sembra essere più semplice e sicura da danni al peduncolo vascolare aberrante. Nonostante la tecnica non sia recente e gli ultimi dati di letteratura siano confortanti, i risultati a lungo termine di questa tecnica video-laparoscopica debbono essere ancora confermati e un attento follow-up è consigliato.

\section{Tecnica chirurgica: pieloplastica laparoscopica robot-assistita}

È abitualmente realizzata per via trans-peritoneale, utilizzando una porta ombelicale da $10 \mathrm{~mm}$ per l'ottica e due porte da 5,8 o 10 $\mathrm{mm}$ come vie operative. Occasionalmente si può utilizzare una ulteriore porta operativa. La tecnica è analoga a quella utilizzata per la pieloplastica laparoscopica trans-peritoneale descritta, ma si adatta preferibilmente a bambini di età superiore ai 4-5 anni, anche se in letteratura sono presenti applicazioni anche nel primo anno di vita. Il grande vantaggio della assistenza del robot è la relativa facilità nella esecuzione della sutura intracorporea. Inoltre, ulteriori vantaggi sono una migliore ergonomia per il chirurgo, una visione tridimensionale, l'eliminazione dei tremori delle mani e un maggiore angolo di curvatura dello strumentario laparoscopico. Come elementi negativi vanno considerati strumentazioni e porte non ancora ideali per piccoli bambini e soprattutto gli elevati costi di acquisto di utilizzo del robot, a tutt'oggi proibitivi per alcuni centri chirurgici urologici pediatrici.

\section{Tecnica chirurgica: endopielotomia}

Una serie di cateteri a palloncino sono stati proposti e utilizzati per la dilatazione o la sezione endoscopica del GPU, per via anterograda da nefrotomia o per via retrograda ascendente, attraverso ureteroscopia o sotto semplice controllo radiologico. Richiede la applicazione di stent pielo-ureterale con JJ. La tecnica ha dimostrato discreto successo nei pazienti adulti con pieloplastiche fallite, mentre non ha dato fino ad ora risultati soddisfacenti nelle idronefrosi congenite da giuntopatia intrinseca in età pediatrica. Non va utilizzata in sospetto di vasi aberranti polari inferiori.

\section{Trattamento post-operatorio e follow-up}

Una notevole variabilità esiste in letteratura sull'uso di stent pielo-ureterali o il non uso di alcuno stent, come anche di drenaggio peripielico e di catetere vescicale, a discrezione del chirurgo operatore, senza evidenza certa di efficacia statisticamente valida. Generalmente uno stent trans-anastomotico è utilizzato, sia esterno nefrostomico, da rimuovere dopo 7 giorni, sia interno tipo JJ, da rimuovere in cistoscopia con pinza da corpo estraneo dopo 4-6 settimane circa. I drenaggi della loggia renale sono da rimuovere abitualmente entro 3-4 giorni, in assenza di secrezioni dalla ferita. La copertura antibiotica è opportuna nei primi 7 giorni, e può essere mantenuta in profilassi a basso dosaggio in caso di stent $\mathrm{JJ}$ interno, responsabile di eventuale colonizzazione batterica e di reflusso.

I controlli strumentali prevedono abitualmente un'ecografia renale a 3, 6 e 12 mesi e una renoscintigrafia sequenziale con MAG3 a 6-12 mesi dall'intervento, ma ogni chirurgo può variare la sequenza dei controlli in rapporto alle proprie preferenze e alla situazione specifica del paziente operato. Un controllo periodico della pressione arteriosa può essere opportuno, specie in casi di ridotta funzione renale residua, per il rischio di ipertensione arteriosa nefrogenica. È spesso consigliato un controllo nefro-urologico completo alla pubertà.

\section{Conclusioni}

Va ricordato che la pieloplastica standard con accesso aperto lombotomico resta una tecnica di elevato successo (95-98\%) nel risolvere l'ostruzione del giunto superiore. Pertanto ogni approccio innovativo, in particolare le tecniche video-laparoscopiche miniinvasive, debbono confrontarsi con essa. Non vi è alcun dubbio che le tecniche minimamente invasive rappresentino un grande vantaggio per il piccolo paziente e per la durata della degenza clinica, specie nei bambini più grandi di età.

Una tecnica intermedia tra quella open e la laparoscopica è rappresentata dalla pieloplastica video-laparo-assistita retroperitoneale (OTAP-OPRAP) che può essere una valida e pratica opzione prima di passare alla mininvasiva pura endocorporea. La pieloplastica laparoscopica dismembered secondo Anderson-Hynes è più impegnativa, specie se con acceso retro peritoneale, garantendo tuttavia ottimi risultati in mani esperte. La tecnica laparoscopica trans-peritoneale del vascular hitch offre ottimi risultati nei casi d'idronefrosi da vasi polari aberranti, purché la selezione dei casi sia rigorosa. La pieloplastica robot-assistita è ottimale nei bambini di età maggiore di quella propriamente infantile, purché ovviamente sia a disposizione la strumentazione ed il personale preparato. Un dato è certo: l'avvento delle tecniche mini-invasive video-laparoscopiche sta attualmente soppiantando la classica pieloplastica con accesso open in un numero sempre maggiore di centri di Urologia e Chirurgia Pediatrica. ${ }^{1-29}$

\section{Bibliografia}

1. Hellstrom J, Giertz G, Lindblom R. Pathogenesis and treatment of hydronephrosis. Paris, France: Societé International d'Urologie; 1949.

2. Chapman TL. Urology in outline. London: Churchill Livingstone; 1959.

3. Dewan P, Clark S, Cendron S, Henning P. Ureterocalycostomy in the management of pelvi-ureteric junction obstruction in the horseshoe kidney. BJU Int 1999 84:366-8. 
4. Janetschek G, Peschel R, Bartsch G. Laparoscopic Fenger plasty. J Endourol 2000;14:889-93.

5. Woodward M, Frank D. Postnatal management of antenatal hydronephrosis. Brit J Urol Int 2002;89:149-56.

6. Wilkinson AG, Rajan P, MacKinlay GA. Endoluminal ballon dilatation for pelvi-ureteric junction obstruction in children: an effective alternative to open pyeloplasty. J Pediatr Urol 2005; 1;301-5.

7. Dhillon HK. Antenatal diagnosis of urinary tract anomalies. 2nd ed. London, UK: Hodder Amold Publishers Ldt.; 2005. Chapter 50.

8. Lima M, Tursini S, Ruggeri G, et al. One trocar assisted pyeloplasty (OTAP): initial experience and codification of a technique. Pediatr Med Chir 2007;29:108-11.

9. Braga LH, Lorenzo AJ, Sheldon S, et al. Failed pyeloplasty in children: comparative analysis of retogtade endopyelotomy versus pyeloplasty. J Urol 2007;178:2571-5.

10. Tanaka ST, Gtantham JA, Thomas JC, et al. A comparison of open versus laparoscopic pediatric pyeloplasty using the pediatric health information system database: do benefits of laparoscopic approach recede at younger ages? J Urol 2008;180:1479.

11. Gundeti MS, Reynolds WS, Duffy PG, Mushtaq I. Further experience with the vascular hitch (laparoscopic transposition of lower pole crossing vessels): an alternative treatment for pediatric ureterovascular ureteropelvic junction obstruction. J Urol 2008;180:1832-6.

12. Valla JS, Breaud J, Griffin SJ, et al. Retroperitoneoscopic vs open dismembered pyeloplasty foe ureteropelvic junction obstruction in children. J Pediatr Urol 2009;5:168-71.

13. Caione P, Lais A, Gerocarni Nappo S. One-port retroperitoneoscopic assisted pyeloplasty versus open dismembered pyeolplasty in young children. J Urol 2010;184:2009-15.

14. Scuderi MG, Arena S, Di Benedetto V. One-trocar assisted pyeloplssty. J Laparoendosc Adv Surg Tech 2011;21:651-4.

15. Peters CA: Pediatric robo-assisted pyeloplasty. J Endourol 2011;25:179-85.

16. Radfort A, Thomas DFM, Subramaniam R. Ureterocalycostomy in children: 12 years experience in a single centre. BUJ Int 2011;108:434-8.

17. Polok M, Chrzan R, Veenboer P, et al. Nondismembered pyeloplasty in a pediatric population: results of 34 open and laparoscopic procedures. Urology 2011;78:891-4.
18. Sakoda A, Cherian A, Mushtaq I. Laparoscopic transposition of lower pole crossing vessels ("vascular hitch") in pure extrinsic pelvi-ureteric junction (PUJ) obstruction in children. Brit J Urol Int 2011;108:1364-8.

19. Cherian A, Nemade H. The "double hitch" manoeuvre in laparoscopic pyeloplasty. Early experience. J Ped Urol 2013; 9:524-6.

20. Lima M, Ruggeri G, Messina P, et al. One-trocar assisted pyeloplasty in children: an 8-year single institution experience. Eur J Pediatr Surg 2015;25:262-8.

21. Silva MV, Levy AC, Finkelstein JB, et al. Is peri-operative urethral catheter drainage enough? The case of stentless pediatric robotic pyeloplasty. J Pediatr Urol 2015;11:175-9.

22. Lee LC, Kanaroglou N, Gleason JM, et al. Impact of drainage technique on pediatric pyeloplasty: Comparative analysis of externalized uretero-pyelostomy versus double-J internal stents. Can Urol Assoc 2015;9:453-7.

23. Badawy H, Zoaier A, Ghoneim T, Hanno A. Transperitoneal versus retroperitoneal laparoscopic pyeloplasty in children: randomized clinical trial. J Pediatr Urol 2015;11:122.

24. Salo M, Altemani TS, Anderberg M. Pyeloplasty in children: perioperative results and long-term outcomes of robotic-assisted laparoscopic surgery compared to open surgery. Pediatr Surg Int 2016;32:599-607.

25. Turrà $\mathrm{F}$, Escolino M, Farina $\mathrm{A}$, et al. Pyeloplasty techniques using minimally invasive surgery (MIS) in pediatric patients. Transl Pediatr 2016;5:251-5.

26. Chiarenza SF, Bleve C, Fasoli L, et al. Ureteropelvic junction obstruction in children by polar vessels: Is laparoscopic vascular hitching procedure a good solution? Single centre experience on 35 consecutive patients. J Pediatr Surg 2016;51:310-4.

27. Esposito C, Bleve C, Escolino M, et al. Laparoscopic transposition of lower pole crossing vessels (vascular hitch) in children with pelviureteric junction obstruction. Transl Pediatr 2016;5:256-61.

28. Chan YY, Durbin-Johnson B, Sturm RM, Kurzrock EA. Outcomes after pediatric open, laparoscopic and robotic pyeloplasty at academic institutions. J Pediatr Urol 2017;13: 49-54.

29. Cobellis G, Nino F, Pierangeli F, et al. Retroperitoneoscopic one-trocar-assisted pyeloplasty in children: an age-related evaluation. J Laparoend Adv Surg Tech 2017;27:651-4. 